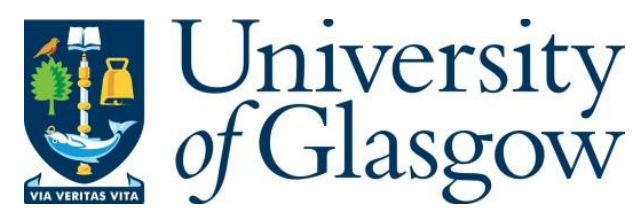

Shah, S. A. and Fioranelli, F. (2019) RF sensing technologies for assisted daily living in healthcare: a comprehensive review. IEEE Aerospace and Electronic Systems Magazine, 34(11), pp. 26-44.

There may be differences between this version and the published version. You are advised to consult the publisher's version if you wish to cite from it.

http://eprints.gla.ac.uk/188135/

Deposited on: 11 June 2019

Enlighten - Research publications by members of the University of Glasgow http://eprints.gla.ac.uk 


\title{
RF Sensing Technologies for Assisted Daily Living in Healthcare: A Comprehensive Review
}

\author{
Syed Aziz Shah, Francesco Fioranelli, School of Engineering, University of Glasgow, UK.
}

\begin{abstract}
The aim of Radio-Frequency (RF) sensing for assisted living is to deliver automatic support and monitoring for older people in their homes, impaired patients living independently, individuals in need of continuous support, and people suffering from chronic diseases that require them to stay in care-homes or at hospitals. RF sensing technologies have the potential to improve the quality of living of elderly people or disabled individuals in need of timely assistance. This paper provides a comprehensive review on three of the most innovative $R F$ sensing technologies for activities of daily living in healthcare sector (namely active radar, passive radar, and wireless channel information \& RFID sensing) and presents some of the open challenges that need to be addressed.
\end{abstract}

\section{INTRODUCTION}

The proportion of people aged over 65 years is increasing worldwide, with various estimations projecting the percentage of over 65 in next 50 years in the European Union [1]. The challenge of aging population is pushing towards novel healthcare provision that evolves from the traditional hospitalbased system, where the patients are treated or monitored for severe health conditions in a controlled environment, to a more person-centric approach where the patients are observed in their homes remotely via modern technology. This home-centric healthcare model can improve the quality of life of different patients suffering from chronic diseases including Parkinson's, dementia, epilepsy, narcolepsy and multiple sclerosis, and enables them to live longer and better by minimizing the disruption to their usual routine and lifestyle. Moreover, providing the traditional levels of hospital-based healthcare for the increasing number of older people would be unfeasible for families and public authorities, hence there is need to find costeffective and reliable solutions based on emerging technologies.

A challenge to overcome is the provision of reliable and continuous monitoring of individuals at risk, while at the same time allowing them to live independently in their homes. In first instance, the monitoring system needs to detect critical events such as falls or other potentially dangerous behavior, and alert family, care-givers, or first responders for prompt help. The risk of dangerous behavior increases with many health conditions such as Freezing of Gait (FOG) Parkinson's disease, wandering behavior in dementia, sleep attack in narcolepsy disease and seizure in epilepsy disease, affecting older individuals. Studies estimate that $67 \%$ of the dementia patients may exhibit wandering behavior as the disease progresses because of the memory loss and weariness [2]. There is a danger that the patient might leave home or fall and injure him/herself. Similarly, a bedridden or frail older patient might develop bedsores due to lack of repositioning and limited mobility on bed. Sleep apnea is a condition where a person suddenly stops breathing for some time while asleep. FOG is a brief, episodic absence or marked reduction of forward progression of the feet despite the intention to walk, and is one of the most debilitating motor symptoms in Parkinson's patients, as it may lead to falls [3]. Narcolepsy is a neurological disorder that affects the control of sleep and wakefulness [4]. Narcolepsy patients might fall asleep while performing daily routine activities. Epilepsy is also one of the neurological conditions where people experience seizures and involuntary body movements, while performing Activities of Daily Living (ADL). The incidence of these diseases increases with aging, together with a general reduction in mobility due to cardiovascular and musculoskeletal problems, with each individual affected by multiple conditions (multimorbidity).

Many technologies in the literature have been presented for patients monitoring. Camera-based sensors are light dependent, require that the subject should remain in line of sight while being observed, and may raise privacy concerns in private rooms (e.g. bathrooms and bedrooms). Ambient sensors such as acoustic receivers, pressure pads, or passive infrared can be disrupted by ambient interference (noise for acoustic or heat sources for thermal infrared) and are expensive to retrofit in home environments. Wearable devices that consist of accelerometer, gyroscope and magnetic sensors can also be used for monitoring daily routine activities and health related parameters, but these must be worn or carried by the monitored subject, with potential issues of acceptance, discomfort and compliance. Nevertheless, both sensor-based and vision-based methods come at high costs thanks expensive devices, deployment and maintenance overhead. Some of the advantages and limitations are shown in Table 1[5].

The use of Radio-Frequency (RF) sensing has been considered in recent years in the context of assisted living, thanks to its contactless monitoring capabilities (i.e. the endusers do not need to wear or carry devices, or modify their daily routine), and to the possibility of leveraging existing communication signals and infrastructure of opportunity such as common Wi-Fi routers. Systems using RF signals for monitoring both ADL and healthcare parameters (breathing and heartbeat rates for example) can be implemented with different approaches. Systems based on Wi-Fi communication can analyze the temporal variations of amplitude and phase of multiple wireless channels (WCI, wireless channel information) to monitor an area of interest and the subjects present. The Wi-Fi communication signal or other wireless systems can be used as illuminators of opportunity in passive radar approaches, where the direct signal from a Wi-Fi router is cross-correlated with the echoes reflected from subjects in the area under test to extract information on their movements [6]. Finally, RF sensing can be implemented as an active radar 
system, whereby suitable waveforms are emitted, and the received echoes are analyzed to characterize locations and movements of the subjects in a monitored area and detect critical events such as falls[6][7]. In this paper, we examine the state-of-the-art research on RF sensing technologies for assisted living and healthcare, and present examples of key applications.

Table 1 - Advantages and limitations of existing technologies [5]

\begin{tabular}{|c|c|c|c|}
\hline Category & Technology & Advantage & Limitation \\
\hline $\begin{array}{l}\text { Sound- } \\
\text { based }\end{array}$ & $\begin{array}{l}\text { Ultrasonic Acoustic } \\
\text { Audio }\end{array}$ & $\begin{array}{l}\text { a. Very sensitive to motion } \\
\text { b. Objects and distances are } \\
\text { typically determined } \\
\text { precisely } \\
\text { c. Inexpensive (audio) }\end{array}$ & $\begin{array}{l}\text { d. Work only directionally } \\
\text { (ultrasonic) } \\
\text { e. Sensitive to temperature and } \\
\text { angle of the target (ultrasonic) } \\
\text { f. Easily be influenced by other } \\
\text { audio signals/noise } \\
\text { g. Prone to false detections }\end{array}$ \\
\hline $\begin{array}{l}\text { Motion- } \\
\text { based }\end{array}$ & $\begin{array}{l}\text { Accelerometer } \\
\text { Gyroscopes }\end{array}$ & $\begin{array}{l}\text { a. No privacy issues } \\
\text { b. High detection accuracy } \\
\text { c. Lower cost }\end{array}$ & $\begin{array}{l}\text { d. Raise physical discomfort issue } \\
\text { e. No direct linear or angular } \\
\text { f. Position information } \\
\text { g. Insensitive to very slow motions }\end{array}$ \\
\hline $\begin{array}{l}\text { Vision- } \\
\text { based }\end{array}$ & Video camera & $\begin{array}{l}\text { a. Effective security measure } \\
\text { b. Maintain records }\end{array}$ & $\begin{array}{ll}\text { c. Interfere with privacy } \\
\text { d. Ineffective in the dark } \\
\text { e. High computational cost }\end{array}$ \\
\hline $\begin{array}{l}\text { Sensor- } \\
\text { based }\end{array}$ & Body-worn sensors & $\begin{array}{l}\text { a. High detection accuracy } \\
\text { b. No privacy issue }\end{array}$ & $\begin{array}{l}\text { c. Expensive devices (sensors) } \\
\text { d. Disturb or limit the activities of } \\
\text { the users } \\
\text { e. Required sensors installation and } \\
\text { calibration }\end{array}$ \\
\hline
\end{tabular}

We aim to present together approaches based on active radar, passive radar using Wi-Fi signals, wireless channel information of Wi-Fi and RFID systems. These are often investigated and discussed by different, separate research groups and communities, whereas there is significant overlap and the potential for mutual exchange of signal processing and classification/analysis techniques.

\section{CHALLENGES IN RF SENSING FOR ADL}

As an emerging approach to ADL and healthcare monitoring, RF based technology has several challenges to overcome in order to provide cost-effective, easily deployable, feasible and efficient solutions. Some of the major issues encountered are outlined as follows:

\section{ROBUSTNESS}

The robustness and accuracy of the RF-based solutions under different, real-life conditions are pressing issues. The majority of these solutions appear to be tested as proof of concept devices in simplified laboratory conditions, hence ensuring robust performances in actual deployment scenarios remains an outstanding challenge. Furthermore, the data collection, processing, and management must be performed in a computationally efficient manner, to reduce the cost of the required hardware and the time to detect critical events such as a fall or stroke. Therefore, there is a need to perform comprehensive validation studies of RF-based systems for ADL in realistic scenarios. This is particularly important when aiming to detect critical events, such as falls, as a high incidence of false alarms of missed detections could compromise completely the perception and adoption of RF-based technologies.

\section{RELIABILITY}

Reliability is one of the most important concerns to establish whether a developed system is applicable in a specific scenario and for a particular set of activities to monitor. The technologies need to be not only reliable and provide resilience to faults and malfunctioning, but also user-friendly so that the assisted elderly individuals can utilize them with ease and comfort. Proper training for elderly people is significantly important for acceptance of these technologies and to avoid the end-users interfere with their proper functioning, and the solutions provided should minimise the impact on their daily routine. RF sensing technologies should help the assisted elderly people in emergencies, keep track of daily routine activities, and should be able to monitor the required vital signs such as heartbeat and respiration rate.

\section{AFFORDABILITY}

Many RF based devices have been developed to reduce potentially the overall cost of healthcare provision, but affordability for individual users and family is a daunting task. The RF sensing for ADL should minimize the deployment cost of the system and allow reuse and relocation of the components being used which in turn reduces the total cost.

\section{ETHICAL CONCERNS}

Preservation of privacy and secure management and access of personal data are major concerns when designing and deploying systems for healthcare. Although RF-based systems do not collect plain optical images as normal cameras do, measures to protect and encrypt the RF data must be put in place. It is important to establish whether data are partially processed at 
the sensor/device side, and the results sent to a data centre, perhaps located remotely, or the whole bulk of raw RF data sent to the data centre for full processing. This has implications on what data amount and format needs to be securely protected. Another possible concern related to RF systems is the level and exposure to EM (electromagnetic) radiation. This is less of a problem for methods based on WCI data and passive radar, as they would leverage on existing Wi-Fi systems that are widely used and accepted. For active radar systems, where an active transmitter needs to be deployed, the majority of work in the literature uses systems emitting low power at non-ionizing frequencies, comparable to those used by communication systems and accepted standards. Nevertheless, careful consideration of potential risks for persistent exposure to low EM radiation must be considered before widespread adoption of active radar technologies for ADL. It is likely that a risk assessment will also account for the risk of missing out in monitoring performance and effectiveness if active systems are not deployed or their power curtailed, hence the need for a compromise.

\section{RF SENSING TECHNOLOGIES}

This section provides background information for some of the most common and widespread non-contact, cost-effective, robust RF technologies for assisted living and healthcare parameters, i.e. radar-based, Wi-Fi-based, RFID-based systems.

Human activity monitoring leveraging radar-based systems, both in active and passive modalities, has emerged in the literature in recent years, and a growing trend has been observed since then [7]. The received signal strength indicator approach exploiting Wi-Fi signals has been used for a few decades but exploiting the amplitude and phase information of multiple wireless channels for activity monitoring is an emerging technique[8]. Despite the rising implementation of RFID with wireless sensor network technology-based healthcare services, few empirical studies have been conducted to assess the potential of this technology within the healthcare sector [9].

\section{RADAR-BASED SENSING}

RADAR is the acronym for RAdio Detection And Ranging. A radar system in its simplest architecture consists of a transmitter that radiates EM signals, transmitting and receiving antennas (the same antenna can be used for transmission and reception), a receiver, and a processor that performs data analysis to identify the properties of object(s) of interest. Radio waves radiated from the transmitter are backscattered by the object(s), providing information in terms of range, velocity, trajectory, and identity of the object. The velocity of objects can be estimated through the Doppler Effect, i.e. measuring the overall shift on the carrier frequency of the radar signal. The identity of objects can be inferred by analysing their micro-Doppler signatures, i.e. the pattern of small modulations on the backscattered radar signal induced by small movements of oscillating or rotating parts of the objects[6] . In the specific case of human monitoring, the micro-Doppler signature are related to the patterns of oscillations of torso, limbs, and head while the person is moving. These patterns are very specific of individuals and particular activities; hence they are very suitable as a tool for automatic classification and monitoring. Radar systems can be divided into two classes.

\section{ACTIVE RADAR}

Active radars are those for which there is a dedicated transmitter and receiver device, which may be integrated in the same system. This requires dedicated hardware and access to available RF spectrum to transmit radar waveforms but has the advantage of full control of the properties of the transmitted signal. The presence and distance of objects can be easily inferred by calculating the time-of-flight of the radar signal along the transmitter-target-receiver trajectory.

\section{PASSIVE RADAR}

Passive radar systems do not have their dedicated transmitter, but exploits a transmitter of opportunity, which can be another radar system (often referred to as hitchhiking radar), or a communication system (e.g. Wi-Fi, DVB-T). The advantage is simpler hardware and no need to allocate spectrum; the disadvantage is no control on the transmitted waveform, which is often not optimized to provide good radar performance. The presence and distance of objects is determined by calculating the delay between the signal directly obtained from the source and the echo received after bouncing off the objects.

The typical signal processing of radar-based RF sensing is presented in figure 1(a), for both active and passive systems. The process starts with an experimental dataset; this can contain measured data, data generated from a numerical or kinematics model, or a combination of the two to increase the size of the dataset. The data are then processed to obtain range information, phase information, or micro-Doppler information. This latter information is extracted by applying proper timefrequency transformation such as Wavelet Transform (WT), Short Time Fourier Transform (STFT) and Extended Modified B-Distribution (EMBD)[7]. Typically, micro-Doppler information has been used for ADL classification, but the other domains of range and phase may also include relevant information. In the next step, numerical parameters known as features, have to be extracted from the data, so that a classification algorithm can be implemented. To reject false alarms and improve performance, data pre-screening and selection of features are applied to select the most relevant subset of data and/or a subset of features. For instance, this step can determine the start and end points of a potential freezing of gait in Parkinson's patient by examining the speed. The amount of data obtained between the two instances will be processed to extract specific features. The last step involves feeding the extracted feature samples into classifiers based on machine learning (ML) algorithms, where part of the samples is used to train the ML classifier, and the remaining data is used to test the performance of the system. Several classification techniques presenting various computational complexity have been proposed including support vector machines (SVM), k-nearest neighbor (KNN), random forest (RF), and recently deep neural networks. Their performance is typically evaluated in terms of metrics such as accuracy, sensitivity, specificity, and their combinations. It is important to mention that several classification algorithms assume that the input feature samples 
are identically distributed. However, this might not be always true for data obtained for different human activities, where specific actions are related to previous activities and affect future actions.

\section{Wi-Fi-BASED SENSING}

The presence of a person moving within an area of interest continuously disturbs a wireless medium, increase signal propagation paths, attenuate the signal, and induce a shift in frequency[10]. Each physical movement generates a unique change in the wireless channel information (WCI). The WCI is used to describe the RF signal propagation properties comprising the distortions generated by human body movements. This has opened doors for research in healthcare domain using WCI and low-cost off-the-shelf devices such as $\mathrm{Wi}-\mathrm{Fi}$ routers and network interface cards embedded in a computer. The typical received signal strength indicator (RSSI) obtained from Wi-Fi signals can be used to identity different human activities. However, this method fails to detect the intricate small-scale body movements due to respiration and heartbeat, since it is susceptible to noise and suffers from coarse resolution. On the other hand, wireless channel information can detect movements at sub-cm level by examining different frequency channels. The general data acquisition and signal processing of Wi-Fi based activity recognition is shown in figure 1(b). The dynamics for acquisition of WCI data for human activity recognition is similar to that of radar-based systems. The data acquisition, signal processing and classification steps are involved for motion detection. Many commercially available Wi-Fi devices enable us to characterize the wireless channel state and received signal strength indicator. However, multipath propagation effects such as reflection, refraction, scattering can affect the performance of RSSI measurements.

Recently, off-the-shelf Intel 5300 network interface card has been used to record the WCI data [11]. The WCI primarily leverages orthogonal frequency division multiplexing (OFDM) symbols to extract amplitude and phase information and reports a group of 30 OFDM subcarriers (30 by 1 matrix). The advantage of using WCI over RSSI is that one or multiple subcarriers can be used for specific application by examining the amplitude and phase information of particular subcarrier(s). The host computer equipped with network interface card connected to the access point (Wi-Fi router) through wireless medium constantly receives the WCI packets. The raw phase data received is extremely random and unusable, hence phase sanitization function is performed on raw WCI samples to obtained useful phase information. Continuous or periodic WCI measurements as a function of time enable us to examine the

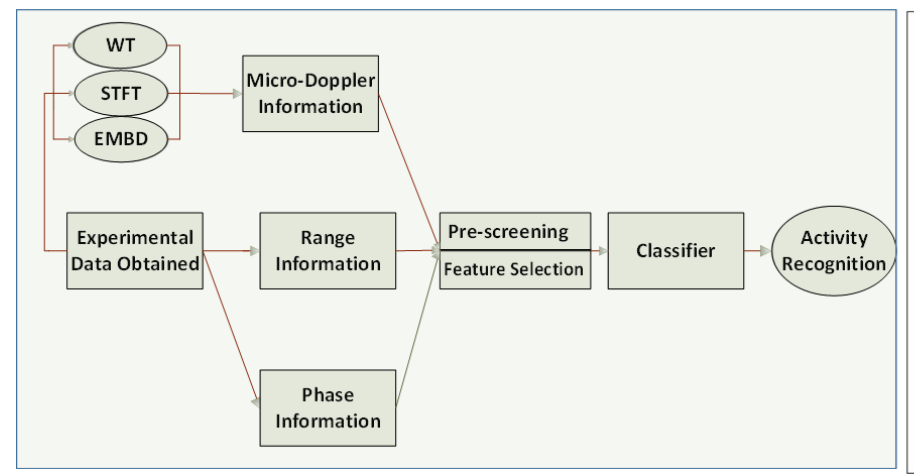

direction, the physical change in individual's position, and the angle of arrival. This is due to the disturbances in the wireless channel over time created by the presence of people and their movements. If a person stands still within $\mathrm{Wi}-\mathrm{Fi}$ range, the small-scale body movement of torso or limbs will distort the wireless medium, and as a result changes in the amplitude, phase, frequency will be observed. As an example, typical daily routine activities made up of different body motions from different body parts, can lead to distinct temporal WCI signatures[11]. Each human body motion induces a unique WCI imprint considering the variances of amplitude information. Each subcarrier is examined, and one subcarrier is selected for specific analysis. The amplitude and sanitized phase information of selected subcarrier(s) are then classified using machine learning techniques to classify different body movements [11].

\section{RFID-BASED SENSING}

The radio-frequency identification technology is also widely used for monitoring and identification tasks. The first RFID identification principles were introduced in [12] to identify friend or an enemy. In today's age, the RFID tags are compact, complex and are able to perform various tasks. The RFID tag consists of an integrated circuit embedded in components including RF, analog or digital circuits. The complexity of RFID is capable to perform many functions and data processing routines that allow the application and deployment of the technology in different areas.

The RFID technology identifies objects through the tags deployed on objects' bodies, hence there is no need for the tags and tag reader to be in line-of-sight. The technology only relies on the wireless communications between tag and tag reader. The RFID system consists of three main components.

\section{CASE STUDIES}

The challenge of human activities recognition for assisted elderly living in healthcare sector include fall detection, vital signs such as respiratory symptoms monitoring, sleep attack detection, gait monitoring and body posture recognition on bed[10]. This section presents case studies in support of practical experimental results to demonstrate that radar-based systems, Wi-Fi-based systems exploiting WCI and RFID-based systems can be used to identify various human movements for ADL considering various aspects of the technologies.
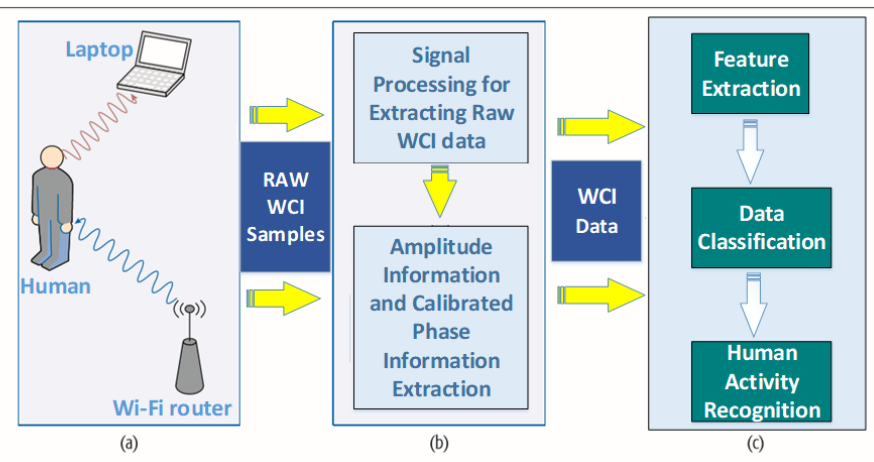
Figure 1 - (a). Data processing for radar-based system to detect human activity. (b). The general structure for Wi-Fi based human activity recognition system.

\section{RADAR SENSING FOR HEALTHCARE}

The idea of remotely monitoring physiologic functions in humans via radars started as early as the 1970s [13] but the development was limited due to the cumbersome and expensive apparatus used in those years [14]. Past few decades have witnessed new baseband signal processing methods and system-level technologies in the context of radar technology for healthcare applications in order to improve the detection accuracy and robustness. Numerous advantages of noninvasive detection and monitoring using radar technology include energy optimization in smart homes, monitoring activities of daily living, fall detection, tumor tracking and so on. Recent advancements in radar technology in healthcare sector have open doors for several human activity monitoring applications in indoor settings[15][16][17]. The radar technology has not only enabled us to monitor heart rate and chest movements but also provides significant information about body structure and orientation, torso displacement and speed of human subjects moving within radar range. The measurements obtained can be used to examine specific types and behaviors. For instance, sleep is considered to be one of the key parts in one's mental and physical health. The quantity and quality of sleep an individual observes have significant impact on safety, mood, immune system, memory and learning. The doppler information obtained using a radar illustrate adequate and non-intrusive detection of one's sleeping pattern. In some cases, the doctors might want to examine the breathing rate of patients while asleep. SleepSense [18] is cost-effective and noncontact Doppler radar-based respiratory monitoring system. The method identifies different sleep postures on bed including sleep-wake cycles and breathing patterns. It was implemented by extracting different time-domain and frequency-domain features, and a decision-tree based machine learning algorithm was applied to identify different sleep stages including breathing, sleep-wake cycles and movements on bed. The effectiveness of the proposed solution was demonstrated for 75minute case study and presented an accuracy of $94 \%$ when breathing rate was extracted.

Radar technology also provide a low-cost, effective and noninvasive solution for personal identification without use of expensive cameras [19].

\section{RADAR FOR ACTIVITY CLASSIFICATION}

The use of radar technology for activity classification in indoor settings require a comprehensive and robust integration of various techniques such as signal processing, RF sensing and artificial intelligence (i.e. machine learning and deep learning. Some of the main applications of radar for healthcare applications is described in figure 2 .

\section{RADAR SENSING TECHNIQUE}

Several short-distance radar systems have been introduced for various applications including continuous-wave $(\mathrm{CW})$, Ultrawide band (UWB) radar, pulsed radar and frequency modulated continuous wave (FMCW) radar. Typically, radars are classified based on operating frequency i.e. C-band, X-band, K- band and so on. The directivity is also one of diversities of radar sensing and is primarily introduced by antenna design and structure. However, the sensing principles almost remain the same, irrespective of the type of radar used.

Radar sensing allows us to obtain data which carry information in determining the target's position, speed and other characteristics. The data obtain requires demodulation using specific algorithms. The acquired data after demodulation needs to be stored in matrices or vectors. For example, having a series of sinusoidal signals over long period of time, one might be interested in extracting information such as amplitude, phase and frequency of the signal rather than analyzing each point of time series. The extracted information can be statistical or multidomain. Some of the popular statistical features are mean, standard deviation and variance. Other commonly techniques used to extract information are discussed as follows:

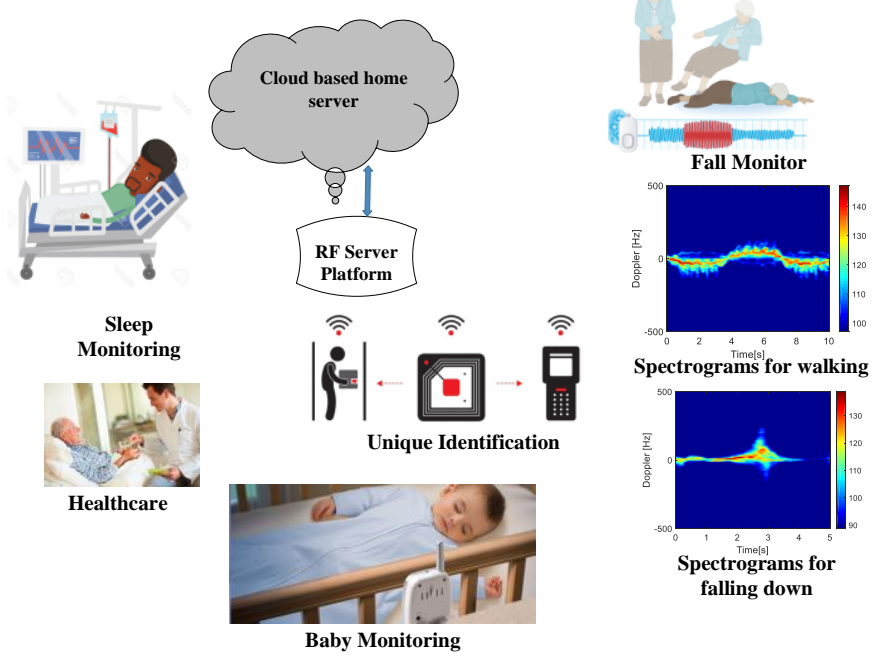

Figure 2 - Radar activity recognition for healthcare

\section{RADAR APPLICATIONS}

The radar sensing has wide range of applications in healthcare sector by continuously monitoring people within its range[21]. Continuous sensing of chest movement and heartbeat is applicable in many areas including safety and security, military and healthcare. Several papers have been published focusing on accurately measuring the respiration rates[22][23] and the instantaneous vital sign rates, which are related to physiological dynamics, are important indicators of human health condition [24][25]. On the contrary, identifying other body movements such as human body orientation is still a challenging task. The change in phase information obtained from Doppler radar is directly related to the chest movement of a person, signal power due to reflection by human body depends on the wave encountering the person's body. The total signal back-scattered due to moving parts of a person can be easily characterized by the Doppler radar cross section (RCS). The radar cross section is directly proportional to the physical area covered by the chest in conjunction with the reflectivity of the surface and directivity of the specific radar at specific operating frequency. 
Furthermore, various types of body movements on all sides and asymmetry of the human body along vertical axes, the chest orientation either on horizontal axis or vertical axis and transmitter-receiver pair influences the radar cross section. Some of the applications of radar for healthcare are discussed as follows:

\section{POSITION RECOGNITION ON BED}

One of the simplest and effective methods to detect the sleep position on bed is presented in [26]. The work primarily presented different types of sleep position on bed using the RCS and it was reported that the RCS for the back side of the person was approximately nine times greater than the person's frontal side. The I-Q component for the back-scattered are shown in figure 3 [26].

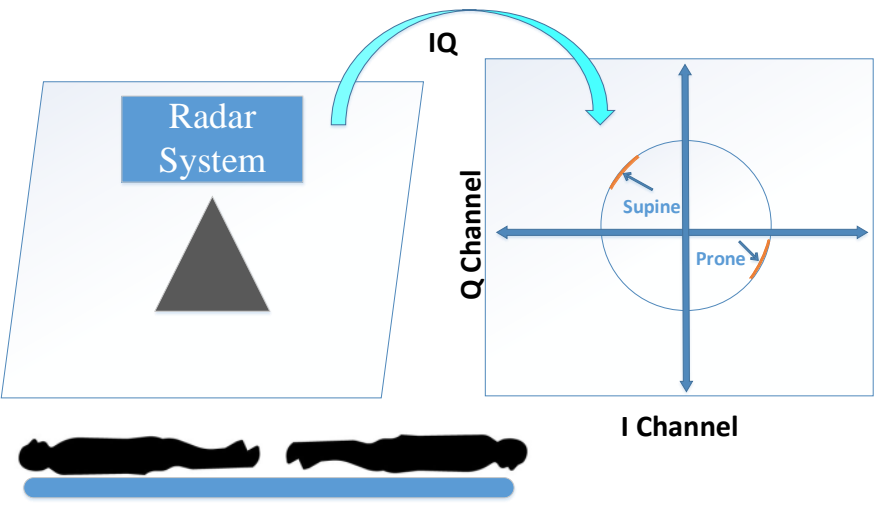

(a)

(b)

Figure 3. (a). Person's breathe detection in supine position (b). Respiration rate detection in Prone position [26].

In order to estimate the RCS by eliminating the direct current (DC) component, the matrix containing complex values were divided into multiple segments in such a way that every segment contained full respiration cycle. The value of the radius for each arc was then found out by employing the center approximation as in [27].

\section{PATIENTS SUFFERING FROM SLEEP CONDITIONS}

Patients with sleep disorders or sleep conditions have typical abnormal sleeping patterns that can lead to emotional, physical and mental disorders. Studies show that there are several kinds of sleep disorders. Polysomnography (PSG) is widely used to study and monitor patients with sleep conditions. The PSG is used to monitor muscle activity (EMG), eye movement (EOG), brain activity (EEG) and heart rates (ECG). Patients using this technology has to spend a night in a specialized lab and all the physiological markers are obtained using wearable sensors which make the solution for monitoring abnormal sleep episodes inconvenient, complex and highly expensive. A noninvasive physiological parameters recording radar known as physiological monitoring radar system (PRMS) was introduced in [28]. The PRMS system is cost-effective, convenient and efficiently monitors the sleep cycle of a person. This solution for real-time monitoring of sleep apnea condition used $\mathrm{CW}$ radar.

\section{UNIQUE-IDENTIFICATION OF INDIVIDUALS}

Each person has a unique breathing pattern and is slightly different from another in terms of expiratory direction, lungs movements due to air influx, tidal volume and so on. Apart from these diversities, the recording of breathing patterns in ventilation at rest when person is in steady-state condition, normal breathing condition and abnormal breathing episodes are examined in ventilatory chambers [19]. This variable condition has a unique pattern and may be due to neural system or due to electro-chemical loops. It is obvious that each person presents a specific breathing pattern amongst innumerable combination of airflow and ventilatory profiles [19]. A large set of data obtained for hundreds of people examined over a period

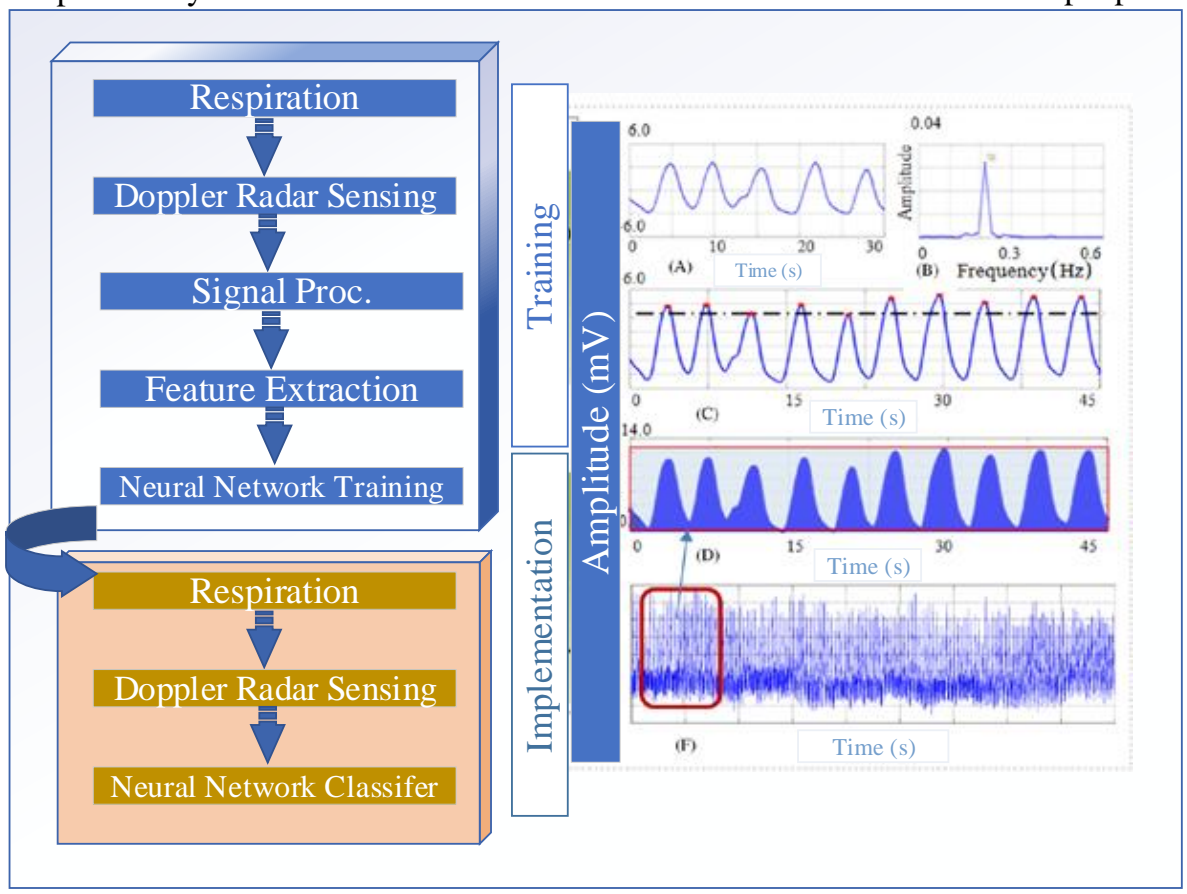

Figure 4. General architecture for identifying people based on vital signs. 
of time, these profiles might lead to a unique identification of person based on their vital signs acquired which can potentially give another aspect to the conventional fingerprinting system. A unique-personal identification system was introduced by Rahman et al. [19] which identifies people based on their vital signs using a continuous wave radar system. The general architecture of the proposed system having training and implementation stage is shown in figure 4.

\section{OCCUPANCY MONITORING USING RADAR}

Distinguishing multiple people in indoor environment using radar system is a challenging task. However, leveraging multiple antennas for radar technology, the sensor might be able to count people and distinguish them using various cardiovascular imprints irrespective or identifying life within the area of interest. The occupancy monitoring using radar is possible by examining the heart rate of people present. Using angle of arrival, very similar heart rates can be detected using single antenna, multiple antennas, single input and single output, and multiple inputs and multiple outputs. Estimating the angle of arrival of signals can help in separating and classifying people spatially in a multiple antenna environment. An S-band quadrature receiver based on Doppler radar using coaxial components was used to detect the people within area of interest [29]. The core idea of this work was to build an efficient and alternate way to tune devices turn off when they were not being used. Therefore, the proposed work saved large amount of electrical power which made it into a sustainable and long-term solution for occupancy monitoring.

\section{DAILY ROUTINE ACTIVITY RECOGNITION}

Activity recognition and estimation of gait parameter are medically essential components of remote health monitoring systems that can improve quality of life, enable personalized treatments, acquire continual medical data to better inform doctors of the patient well-being, reduce health costs, and ensure rapid response to medical emergencies[30].The radar sensing technologies can be used to detect different kinds of gaits by extracting the range, Doppler, amplitude, and phase information[31]. These capabilities enable to detect daily routine activities and classification of human gait [32] such as walking, sitting and standing, squatting, bending down to grab objects, walking while carrying a box and critical events such as fall [33][34]. These relatively simple actions can potentially be combined to detect macro-activities such as food preparation, getting dressed, or house cleaning, which can be used to monitor the wellbeing of people. Regular performance of these activities can be considered as a sign of good health, whereas anomalies may be used as an indication of degrading health conditions. $\mathrm{Li}$ et al. [35] proposed a multisensory approach to investigate daily routine activities using radar sensors in conjunction with wearable devices to enhance the classification accuracy. A Frequency Modulated radar sensor operating at $5.8 \mathrm{GHz}$ frequency was used. The experimental environment was an office room at the University of Glasgow as described in [35]. Ten different volunteers participated in the experiment and were asked to repeat each activity three times. The activities include walking with box, walking without box, sitting on chair, standing up, bending down, bend-down and tie shoe lace, drinking water, taking a phone call, simulate a frontal fall on a mat, and bending down on knees followed by coming back up. Similar activities were including deliberately to validate the robustness of the proposed classification algorithm. Information fusion techniques combining radar sensor and wearable sensors were presented to overcome the limitation of using one sensor for activity recognition. The overall accuracy of the proposed solution improved from $80 \%$ to $98 \%$ when multi-sensory approach was adopted. Another example of radar sensing for activity recognition is in [36]. This work focused on the identification of three kind of human gaits, i.e., walking without carrying hand-bag, walking with holding a hand-bag

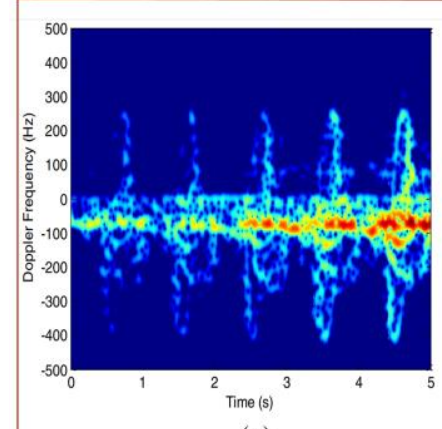

(a)

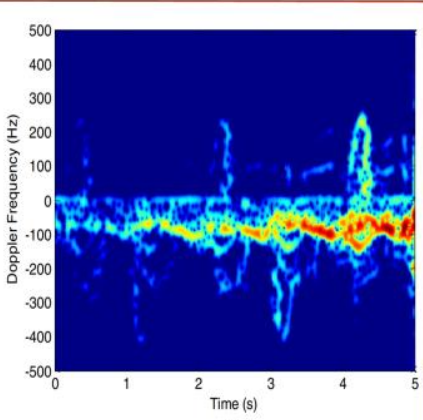

(b)

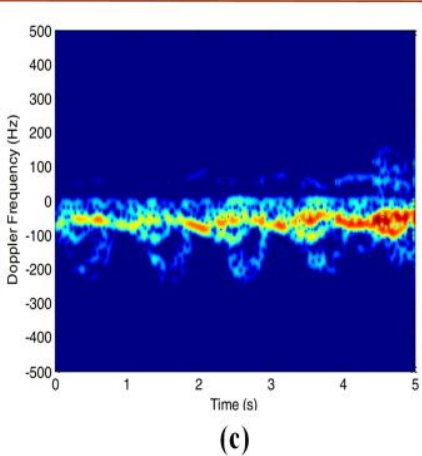

(c)

Figure 5. Spectrograms of human gait - (a). Walking with no hand-bag (b). Walking and holding hand-bag with one hand (c). Walking and hold hand-bag with two hands [12]

with one hand, and walking with a hand-bag holding by both hands, using X-band $(9.8 \mathrm{GHz})$ continuous wave $(\mathrm{CW})$ radar. The primary reason of classifying these three human gaits is because of its usefulness in safety and security applications, as unauthorized items might be carried out in the hand-bag. The human gait recognition using $\mathrm{CW}$ radar involved obtaining data in time-frequency domain using short-time Fourier transform (STFT), extracting micro-Doppler features and applying machine learning algorithms such as SVM for data classification. Two metrics, Doppler offset and bandwidth, were chosen as the two main parameters and were closely associated with swinging of an arm. Figure 6 describes the spectrograms for three different kinds of human gaits obtained during the experiments. Figure 5(a) illustrates the spectrogram for a person with no hand-bag. The two arms of the person move back and forth freely while walking. When the person was carrying the hand-bag with one hand, one arm would swing back and forth while the other arm was more constrained by the item carried. Hence, the peak values of positive and negative 
signature appeared alternately in the micro-Doppler as shown in figure 5(b). When the person was walking and holding the hand-bag with two hands, the majority of the time-frequency components would come from torso and legs movements, generating only small peak values for this third gait as seen in figure 5(c). The data obtained was then processed through SVM to analyze the accuracy of the proposed model and it was observed that high classification accuracy was achieved considering the three human gaits.

\section{Wi-Fi-BASED SENSING FOR HEALTHCARE}

For past five years, the RF sensing leveraging Wi-Fi technology have witnessed exponential growth across many top-tier institutions. For instance, Yang et al. [3][37][38][39][40][41] have introduced several application of Wi-Fi sensing for healthcare applications. Moreover, $\mathrm{Pu}$ et al [42] proposed a WiSee protype that can control smart devices using human gestures. Different ground-truth protypes and models for different applications have been introduced mainly focusing on the small-scale body motion identification and classification, activities of daily living, vital signs monitoring, indoor localization and so on[43][44]. The experimental results obtained using Wi-Fi sensing indicate high accuracy can be achieved for specific purposes. Due to increase in the adaptation of the Wi-Fi sensing technology, some of the most common applications are discussed as:

\section{Wi-Fi-BASED KEY STROKE IDENTIFICATION}

A keystroke recognition system leveraging channel state information obtained using Wi-Fi signals was proposed by Ali et al [45]. The authors, for the first time demonstrated that wireless channel information can be used to identify keystrokes of 37 available keys on keyboard such as 1 space bar, 10 digits and 26 alphabets. The authors exploited the finger and hand movement which carry unique signature in terms of formation and direction when the user is typing specific keys that produce a unique wireless channel imprint as a result generates specific CSI values known as CSI-waveforms. This method uses the induced CSI values for each keystroke in their feature space.
The state-of-the-art Wi-Fi devices transmits data at higher data rates and are enough to form a high-resolution CSI-data for specific keystroke. The Principal Component Analysis (PCA) was used to eliminate the unwanted noisy components from the signals received. In real-world application, this method can identify keystrokes continuously and can determine the sentences with an accuracy of $93 \%$. However, the proposed framework only works in a controlled setting and has several limitations.

\section{FINGER-GRAINED GESTURE RECOGNITION}

The finger-grained gesture recognition or Wi-Finger proposed in [46] obtained number and text as input using finger-grained gestures of 9 digits using commercially available, low-cost $\mathrm{Wi}$ Fi devices. The core idea was to apply the number and text input for human-compute interaction. The proposed system involved three main stages. Initially, a Hampel identifier was used to remove the outliers. Secondly, a low-pass filer such as Butterworth filter was applied in order to eliminate frequencies ranging between $1 \mathrm{~Hz}$ and $60 \mathrm{~Hz}$. Lastly, a weighted-movingaverage technique was used to further remove unwanted components form the CSI data. The CSI data received was divided into small chunks to extract the desired features for training and testing. The finger-grained gesture recognition leveraging Wi-Fi technology works well for single person under stable environment (where no external movement is observed) including laboratories where only two occupant one subject and another taking reading is present. This system achieved an average accuracy of more than $90 \%$. ransmitter and an omni-directional antenna as receiver. The distance between the transceiver pair was 7 meters. As an example, the variances of amplitude information for three wandering behaviors are presented in figure 7(c). Each gait induced a unique signature, i.e. for random behavior the amplitude information varied between $3 \mathrm{~dB}$ and $25 \mathrm{~dB}$, for pacing the amplitude information fluctuated around $25 \mathrm{~dB}$ and for lapping the data changed between $23 \mathrm{~dB}$ and $25 \mathrm{~dB}$ for subcarrier number 20. A support vector machine was used to classify three different gaits, with an overall accuracy of $90 \%$.

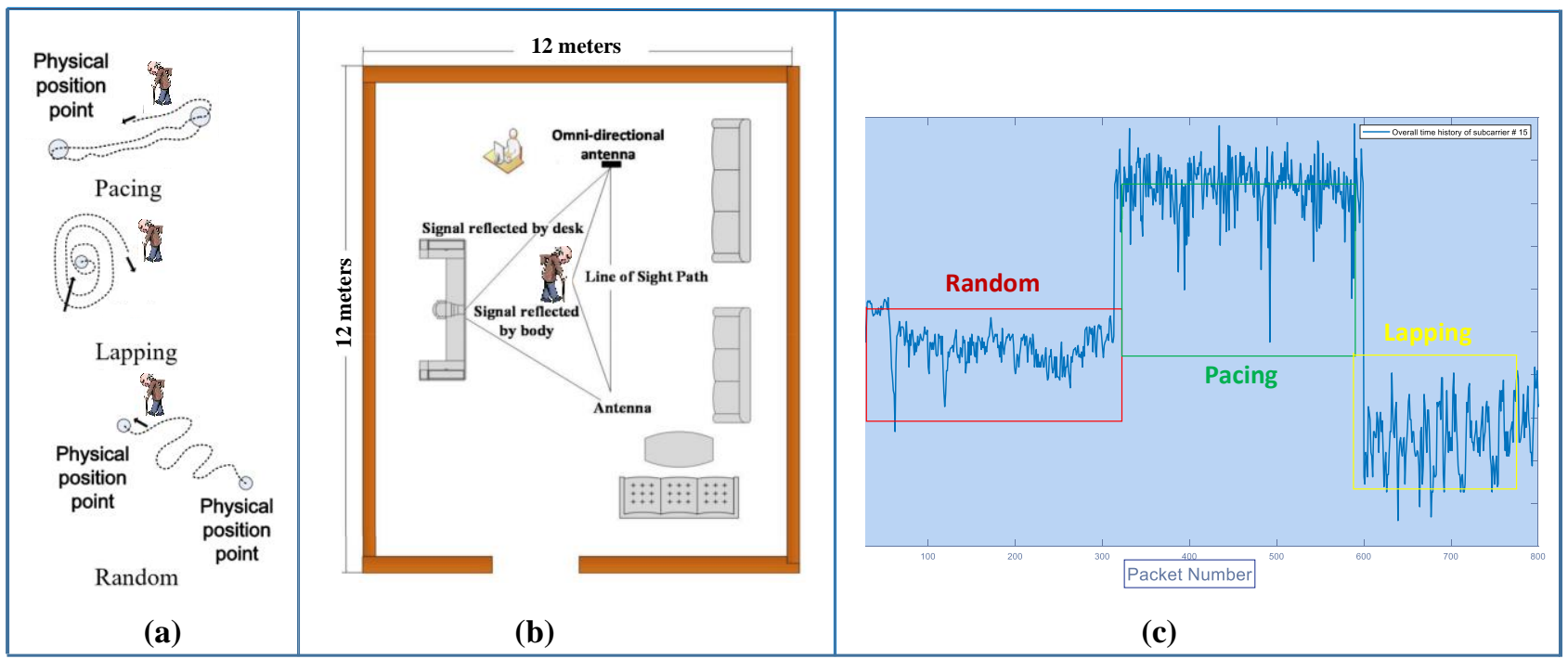

Figure 6. A sketch for three wandering behaviors - (a). Typical scenario for random, pacing and lapping gaits (b). Experimental setup (c). Time history of three gaits considering amplitude information 


\section{Wi-Fi-BASED HAR}

A non-invasive, low-cost indoor human activity recognition system was introduced by Wang et al. [47] known as the E-eyes. The system exploited Wi-Fi signal using channel state information obtained for a group of 30 subcarriers in indoor settings[48][49]. The system provided an adequate solution where minimum wireless network infrastructure was required using commercially available, low-cost, off-the-shelf Wi-Fi devices. Contrary to the gesture identification system, the HAR required loosely defined set of motion over time, generally known as large-scale body movements. As far as data processing for E-eyes is concerned, a dynamic smoothing filer was used to remove high frequency noisy signals and maintain the information in CSI data stream for all activities performed in indoor environment. The proposed system for HAR has two main parts: (a). Profile construction and human activity recognition. During HAR, the CSI data stream are split into its associated activity and variations observed in its movements. The experiments for E-eyes was performed in two indoor settings as shown in [47] and achieved overall accuracy of more than $96 \%$. A similar work on gait recognition was done in [39] where three typical wandering behaviors experienced by dementia patient were monitored using WCI data exploiting Wi-Fi signals. The wandering behaviors include random, lapping and pacing movements as in figure 6(a).

The experiment was performed in a $12 \mathrm{~m}$ by $12 \mathrm{~m}$ room at Xidian University, China as shown in figure 7(b).

\section{VITAL-SIGNS MONITORING USING Wi-Fi SIGNALS}

The breathing pattern causes the chest walls to move by $0.5 \mathrm{~cm}$ to $2.0 \mathrm{~cm}$. This small-scale chest displacement will produce a $0.25 \mathrm{~cm}$ to $1.0 \mathrm{~cm}$ radians phase change at a carrier frequency of $2.4 \mathrm{GHz}$. This allows us to leverage wireless channel information using $\mathrm{Wi}-\mathrm{Fi}$ signals to provide a non-invasive respiratory monitoring solution. The experimental settings and results in [50] demonstrate that the detailed amplitude information carrying breathing pattern can be easily extracted from raw WCI data using low-cost small wireless devices with the help of median filter as shown in figure 8 . The primary purpose of this filter is to remove random noise. The authors have termed the proposed method as S-Band sensing technique (because of the devices operating at $2.4 \mathrm{GHz}$ ). The solution not only presents the subject's normal respiratory pattern, but also provides the abnormal breathing conditions such as bradypnea (abnormal slow breathing) and tachypnoea (abnormal increase in breathing rate). A total number of five volunteers participated in the experiment and each time high classification accuracy was achieved.

\section{SEIZURE EPISODES AND HAR}

Recently, off-the-shelf Intel 5300 network interface card has been used to record the WCI data [51][52]. The WCI primarily leverages orthogonal frequency division multiplexing (OFDM) symbols to extract amplitude and phase information and reports a group of 30 OFDM subcarriers (30 by 1 matrix). The advantage of using WCI over RSSI is that one or multiple subcarriers can be used for specific application by examining the amplitude and phase information of particular subcarrier(s). The host computer equipped with network interface card connected to the access point (Wi-Fi router) through wireless medium constantly receives the WCI packets. The raw phase data received is extremely random and unusable, hence phase sanitization function is performed on raw WCI samples to obtained useful phase information. Continuous or periodic WCI measurements as a function of time enable us to examine the direction, the physical change in individual's position, and the angle of arrival. This is due to the disturbances in the wireless
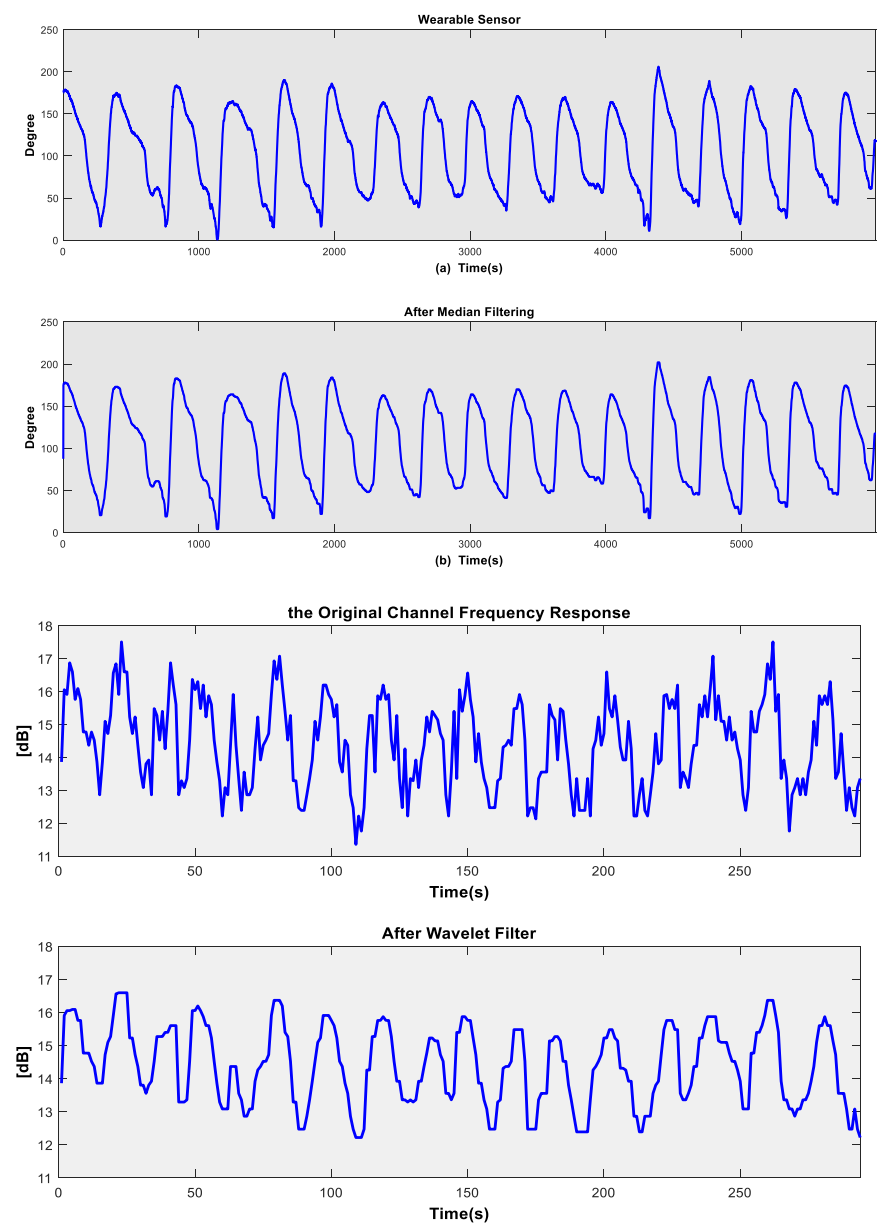

Figure 7. Comparison of breathing pattern recorded using wearable sensor and wireless channel information

channel over time created by the presence of people and their movements. If a person stands still within Wi-Fi range, the small-scale body movement of torso or limbs will distort the wireless medium, and as a result changes in the amplitude, phase, frequency will be observed. As an example, typical daily routine activities made up of different body motions from different body parts as in figure 8, can lead to distinct temporal WCI signatures [53]. We can clearly notice that each human body motion induces a unique WCI imprint considering the variances of amplitude information. Each subcarrier is examined, and one subcarrier is selected for specific analysis. The amplitude and sanitized phase information of selected subcarrier(s) are then classified using machine learning techniques to classify different body movements [54][55].

\section{RFID TECHNOLOGY IN HEALTHCARE SECTOR}


According to a report published by World Health Organization (WHO), the human and medical equipment in hospitals result around 42 million adverse events each year and presents a critical compulsion in order to improve the patient health and safety[56]. The RFID technology has great potential considering the healthcare and wellbeing of patients suffering from diseases by reducing the errors of human-computer interactions. Contrary to its advantages, the technology has been under-utilized in hospitals and care-centers due to difficulties in deployment and investments [57]. Literature review indicate large number of applications of RFID technology in healthcare sector. Some of the applications include person monitoring, identification and tracking, drug detection, blood transfusion, asset and equipment tracking, recording of sensor-driven data [58][59]. For patient health, wellbeing and safety, we have identified four primary application of RFID technology including patient identification, tracking, and patient monitoring.

\section{RF FOR PATIENT IDENTIFICATION (RFPID)}

The hospital and care-center staff on daily basis face challenges such as patient's misidentification which is one of the main reasons for errors medical equipment and is raises high level of risk to patient health and safety [60]. The RFPID technology include wearing wristband equipped with passive RFID tag which can scan patient's identity and store information such as name, date of birth, allergies, blood-type and so on [61]. Numerous studies conducted suggest that hospitals and carecenters such kind of errors by deploying an adequate identification system which can bring improvement in health and safety procedures[62]. Similarly, the work in [63] indicate that the smart RFIDs can minimize errors which are the primary source of misidentification of patients. Other studies in literature as in [57], demonstrate that the identification of patients using RFID technology has a huge potential to acquire accurate medical data from different healthcare systems that can minimize errors to great extent.

\section{RFID FOR TRACKING PATIENTS}

The supply chain management, identification and monitoring of healthcare inventories and assets common applications of RFID in healthcare domain [64]. The RFID technique is now going through a transition period, the period where it was only used for identification, and now viewed as an alternative solution for safety and securing by tracking vulnerable patients admitted in hospitals. The tracking applications of RFID include remotemonitoring of elderly people [65], patients with disorientations [66]. In emergency situations, the RFID technology can be used to locate and identify specific patients to provide immediately medical and health assistance as demonstrated in [67].

\section{RFID FOR PATIENT MONITORING}

Another useful application of RFID in conjunction with other sensors, is to monitor patients with different diseases as introduced in[66] where implantable RFID chip was used as a portable medical record. The RFID technology integrated with mobile networks [68] and sensors can help in detecting critical events such as falls or breathe abnormalities [69]. The Internetof-Things (IoT) and RFID-enabled real-time monitoring of patients was introduced in [70]. Furthermore, Friesen et al.[60] introduced RFID driven smart bandages used to monitor patient's post-surgery status via identifying damaged tissues and detecting body temperature for healing process. A paper published by Amendola et al[71] revealed that the applications of RFID technology for healthcare are still in experimental stages, however recent advancement have witnessed scientists and engineers paving the way print-ink-on-skin like electronic devices used for monitoring patients through wireless medium for a specific period of time.

Moreover, an RFID-based vital signs monitoring system was introduced by Omar et al. [72] and was named 'Smart Patient Management, Monitoring and Tracking System Using RadioFrequency Identification (RFID) Technology'. The primary goal of this system was design and develop a reliable and lowcost system for patients' vital signs monitoring including heart rate, pulse rate, oxygen saturation and temperature, tracking and data management. The system exploited microcontroller board namely Arduino mega 2560 to acquire and pack the sensors data and send it using Wi-Fi communication system as shown in figure 9.

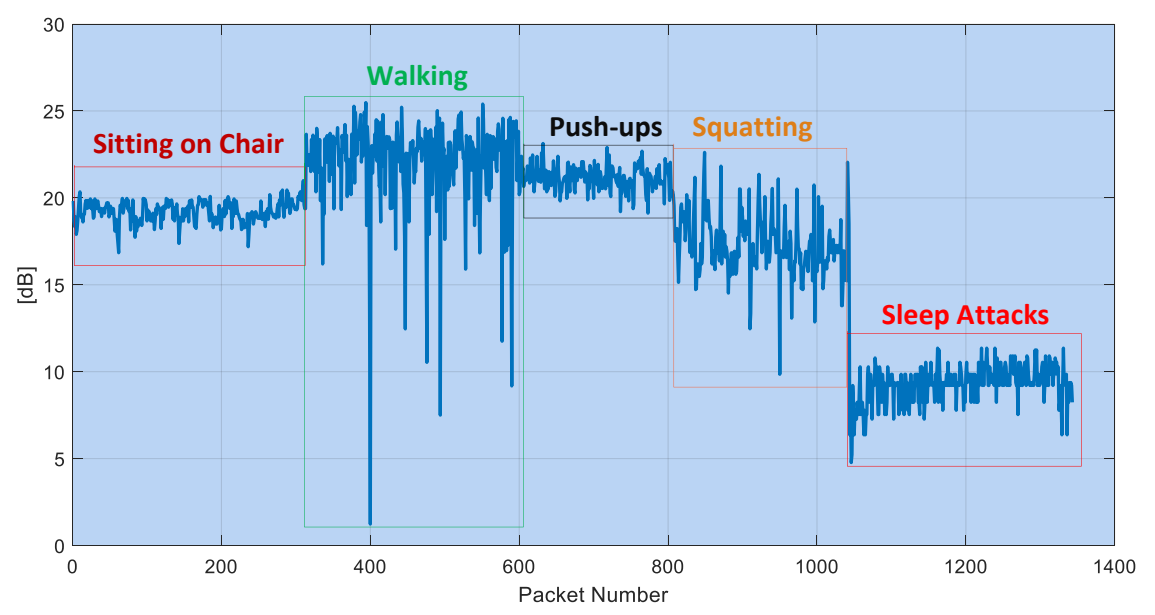

Figure 8. Typical daily routine activities and sleep attack detection exploiting wireless channel information [51] 


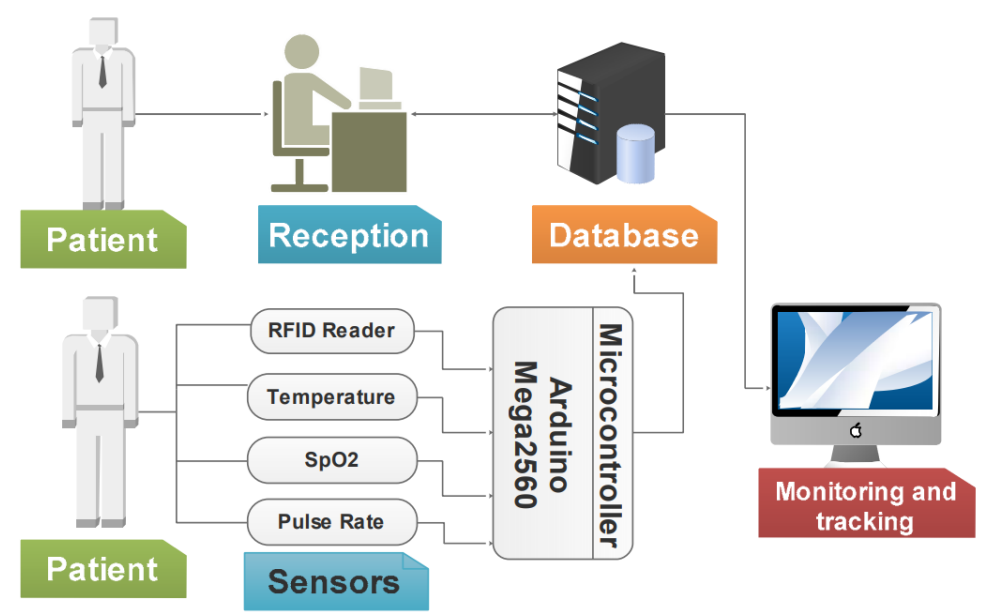

Figure 9. Architecture of RFID-based tracking system

Vora et al [73] introduced vital signs monitoring system capable of detecting normal and abnormal breathing rate using passive RFID tags. This work presented that the identification of the two conditions (i.e. sleep apnea and bradycardia) are possible using RFID. It was demonstrated that rate showed a correlation of $99 \%$ with the actual heart rate when compared to respiration rate tags. The sleep apnea condition with a minimum of 10 seconds threshold was detected. Furthermore, another study conducted in[74] used microsensors integrated RFID chips to sense the vital signals such as pulse, temperature, heart beat and blood oxygen level. The idea was to reduce the potential risk of life taking condition in infants, known as the Sudden Infant Death Syndrome (SIDS). The RFID chips were connected to a mobile phone over wireless link to alert the caregiver or doctor. However, no discussion was made on the performance of the system.

\section{RFID FOR FALL DETECTION}

Falling is one of the major health threats to the independent living of elders, and falling has received much attention in academia and industry over the past two decades[75]. Many systems have been introduced in the healthcare sector to detect fall events in elderly patients. The authors in [76] presented a fall detection system comprising a server for data management and different RFID readers and multiple RFID sensor tags. This system was implemented in such a way that the nurse/caregiver would receive an alert in case of fall events. The proposed method was based on the change in the power level for different human activities and no qualitative results were discussed. Another fall detection prototype based on RFID technology where the design and implementation of a motion detection system based on passive radio frequency identification tags known as TagCare was presented in [75]. The main findings of this work were Doppler frequency was exploited to detect human activities and predict fall event. Extensive experiments were performed to demonstrate the validity and efficiency of TagCare. It was observed that this solution presented an accuracy of more than $96 \%$ for detecting incidents such as falls. Hiroto et al. [77] presented an anomaly detection frameworks based on Ultra High Frequency (UHF) passive RFID that used the fluctuations in the RSSI between the RFID pair of tags. The experiments were performed in indoor environment and each time an accuracy of more than $98 \%$ was achieved. Similarly, various fall detection systems based on RFID were introduced in [78] [79][80].

\section{RFID FOR VITAL SIGNS MONITORING}

Vital signs are a group that show the present status of the human body's vital functionalities. An intelligent infant healthcare solution using active RFID tags was proposed in [81] to reduce some potential risks. This healthcare system could be deployed in in a hospital room, leveraging micro sensors integrate RFID used to detect patient's vital signs including breathing and blood pressure. Moreover, an unconventional battery-free and wireless infant heart and respiration rate monitor that uses passive RFID technology was introduced in [73] . The patient's heart rate data was transmitted using an RFID tag and respiration rate was determined by monitoring the variation in the RSSI obtained from the RFID tag. This healthcare solution reduced the hardware overhead and made possible the continuous monitoring life threatening conditions such as sleep apnea and bradycardia of an infant baby. In order to monitor the blood pressure, heart rate and respiratory pattern, an integrated vital signs monitoring system was proposed in [82]. Exploiting a smartphone in conjunction with RFID chips, all the collected signals can be exported to a server. The microcontroller has EEPROM memory for recording all measured results continuously. This healthcare solution improved the person's data collection procedures by decreasing the processing time and increasing the overall accuracy of the system. The results obtained for vital signs indicated that the percent of accuracy was around $90 \%$.

The RFID applications for healthcare and safety sector are summarized in table 2 .

\section{OUTSTANDING CHALLENES}

There are some outstanding challenges for RF sensing technologies for assisted activities of daily living remain to be incorporated to deploy these systems in real-world scenarios. Specifically, issues related to the use of radar for healthcare application arise from signal processing and deployment perspective. Some of 
the challenges encountered for radar, Wi-Fi and RFID technologies are discussed as follows:

\section{OUTSTANDING CHALLENGES IN RADAR TECHNOLOGY}

One of the biggest challenges is the presence of clutter in indoor settings that may produce multipath effects, as a result false/ghost target. There is also a strong possibility of other people or pets present and moving in the area of interest, thus brining complications to the signatures obtained using radar, resulting in the generation of false alarms. The radar waveforms generated by a radar for healthcare applications has be in compliance with the telecommunication governing bodies, encountering potential limitations in the context of transmitting power and bandwidth utilized, which put constraints on the Signal-to-Noise Ratio (SNR) and range resolution. The probability of detecting human activities and critical events such as falls, irrespective of the sequences of activities performed is also one of the major challenges. A generalize system that is capable of taking the specificity of a subject under observation into account. The specificity may include physical impairment of a patient or any other constraints associated with specific person. This phenomenon makes things complex in order to train a classifier effectively irrespective of observation times and huge data sets associated with a particular subject and environment where the experiments were performed.

The open challenges for using radar technology for ADL can be addressed using multiple radar sensors deployed in indoor settings and may be investigated as a future work including the exploitation of the complementarity of multiple technologies working in parallel. As far as radar technology is concerned, an approach involving multi-static radar systems having various nodes with spatially separated transmitter and receiver capabilities that can cover the whole area in indoor environment. This approach has produced promising performance for micro-Doppler classification in outdoor settings[83][84][85][86][87]. The same approach using radar technology can deliver promising results in detecting fall events indoor environments[88]. A simpler method could be the usage of multiple monostatic radar sensors, instead of using multistatic radar system.

\section{OUTSTANDING CHALLENGES IN Wi-Fi TECHNOLOGY}

On the basis of examining some of the key application of WiFi sensing for healthcare application, all implementation methodologies use the basic principle of RF signal propagating from Wi-Fi transmitter to the receiver through multiple paths. The Wi-Fi signal propagating carries information about particular human activities. In order to put Wi-Fi sensing for assisted activities of daily living from the perspective of realworld application, following open challenges need to be addressed:

All the applications based on Wi-Fi sensing technologies discussed in this paper have provided adequate robustness and accuracy. However, the experiments are only performed in a controlled environment. The results indicate that placement of the equipment's distance between the subject and devices in use, multiple occupant within area of interest and behind-the-wall detection of movement significantly affect and influence the overall accuracy of the system. In this context, the Wi-Fi sensing technology in conjunction with other signal processing technique should be designed in such a way that it should provide reliable and robust performance in real-world scenarios while keep accuracy as high as possible.

- The wireless channel information obtained using WiFi signals can help in achieving recognition tasks using commercially available devices. However, the existing IEEE 802.11n protocol for Wi-Fi signals is the only solution for HAR. Other protocols such as 802.11ac and 802.11ah can be potentially used for healthcare applications.

- Researchers have mostly used low-cost Intel 5300 network interface card for extracting channel state information at the receiver side which only report group of 30 OFDM subcarriers. Other platform such as software defined radios can be used to extract the same information where the number of OFDM subcarriers can be modified as per the requirement of specific application.

\section{OUTSTANDING CHALLENGES IN RFID TECHNOLOGY}

Similar to radar and Wi-Fi sensing for healthcare applications, the RFID based technology also poses several open challenges. Some of the challenges associated with RFID sensing are technological challenges, privacy and security challenges, and organizational challenges.

Technological challenges: The RFID signal transmission can lead to the interference with another electromagnetic signals present within the indoor environment. This potential interference can affect the overall performance and result of medical equipment that bring forward potential thread to subjects safety at hospital or care-center[89]. Hu et al. [90] reported that radiation of RFID tags can have interference with adjacent medical devices including a syringe pump and might cause the device to turn off when it is in proximity with an RFID tag.

Privacy and Security Challenges: Data privacy and security are one of the most important aspect in healthcare applications. In the context of RF sensing for healthcare, ensuring the privacy and security is at top priority when delivery information about a particular subject or patient[91]. The privacy and security concerns stem from accessing the unencrypted data obtained with RFID tags by an unauthorized person.

Organizational Challenges: One of the main organizational challenges in RFID technology is in its deployment. Although RFID tags, these days can be easily implemented due to a significant decrease in its cost. However, the implementation of RFID infrastructure needs databases, servers, middleware and other associated requirements. A study conducted on RFID adaptation reported that governmental policies and environmental barriers are two of the biggest challenges in deploying the technology in developing countries[92]. 
Table 2 - Summary of RFID Applications

\begin{tabular}{|c|c|c|c|c|}
\hline Number & Ref. & Application & Advantage & Limitation \\
\hline 1 & 66 & Safety and Security & $\begin{array}{l}\text { Two stage } \\
\text { authentications }\end{array}$ & Processing overhead \\
\hline 2 & 67 & $\begin{array}{l}\text { Patient Tracking and } \\
\text { Medication Traceability }\end{array}$ & $\begin{array}{c}\text { optimal } \\
\text { configuration of } \\
\text { interconnected } \\
\text { devices }\end{array}$ & $\begin{array}{l}\text { The development and implementation of the system } \\
\text { requires a high economic investment }\end{array}$ \\
\hline 3 & 68 & $\begin{array}{l}\text { Survey on vital signs } \\
\text { monitoring }\end{array}$ & $\begin{array}{l}\text { Comprehensive } \\
\text { overview }\end{array}$ & No gaps and future direction discussed \\
\hline 5 & 69 & $\begin{array}{l}\text { automatic monitoring and } \\
\text { tracking of patients }\end{array}$ & $\begin{array}{l}\text { Hybrid Sensing } \\
\text { network }\end{array}$ & Computational expensive/ deployment overhead \\
\hline 6 & 70 & $\begin{array}{l}\text { Activity monitoring in hospital } \\
\text { environment }\end{array}$ & Multi-modal & No practical implementation \\
\hline 7 & 71 & Body temperature monitoring & $\begin{array}{l}\text { Low-cost, } \\
\text { energy- } \\
\text { autonomous, } \\
\text { and disposable } \\
\text { sensors }\end{array}$ & No discussion on the overall performance and validity \\
\hline$\overline{8}$ & 72 & $\begin{array}{l}\text { Real-time patient monitoring of } \\
\text { vital signs during stay in an } \\
\text { emergency and critical care } 1\end{array}$ & $\begin{array}{c}\text { Generation of } \\
\text { unique } \\
\text { identification }\end{array}$ & Specialized hardware \\
\hline 9 & 73 & Infant vital signs monitoring & $\begin{array}{l}\text { Passive RFID } \\
\text { tags }\end{array}$ & Deployment of tags on infants \\
\hline 10 & 74 & Infant vital signs monitoring & $\begin{array}{l}\text { Multiple infant } \\
\text { monitoring }\end{array}$ & Deployment of tags on infants \\
\hline 11 & 75 & Fall detection & $\begin{array}{l}\text { Leveraging } \\
\text { RSSI+Doppler } \\
\text { frequency } \\
\text { values }\end{array}$ & Tested on only one subject \\
\hline 12 & 76 & Fall Detection & UHF RFIDs & Limited detection range \\
\hline 13 & 77 & Fall Detection & $\begin{array}{l}\text { neighborhood } \\
\text { preservation } \\
\text { principle }\end{array}$ & Reference fall data required for detection \\
\hline 14 & 78 & Tracking and localization & $\begin{array}{l}\text { Pure passive } \\
\text { RFID tags }\end{array}$ & Computational expensive \\
\hline 15 & 79 & $\begin{array}{l}\text { Gait monitoring and fall } \\
\text { detection }\end{array}$ & $\begin{array}{l}\text { passive RFID } \\
\text { tags }\end{array}$ & dual-band RFID module built into a pair of slippers, \\
\hline 16 & 80 & Fall detection & $\begin{array}{c}\text { Multi- } \\
\text { sensor/high } \\
\text { classification } \\
\text { accuracy }\end{array}$ & Computional expensive \\
\hline 17 & 81 & Infant vital signs monitoring & $\begin{array}{c}\text { Blood } \\
\text { oxygen,pulse, } \\
\text { temperature } \\
\text { detection }\end{array}$ & Deployment of tags on infants \\
\hline 18 & 82 & Vital signs & Non-Contact & Specialized hardware \\
\hline
\end{tabular}

\section{ACKNOWLEDGEMENT}

The authors are supported by the UK Engineering and Physical Science Research Council, EPSRC, with grant EP/R041679/1 "INSHEP".

\section{CONCLUSION}

This paper presented a comprehensive review of recent advancements in RF sensing technologies for assisted daily living in healthcare sector. The activity of daily living has become a relevant research field in past few years, as the population of the world is aging, resulting in high number of elderly people in a society. This aging population poses a major challenge in terms of health and wellbeing, and to economy. 
The RF sensing technologies provide robust, low-cost and noninvasive solutions to continuously monitor elderly in indoor settings without disrupting their daily living. In this context, we have discussed three main the technologies such as radar, WiFi and RFID, their advantages and limitations, and their contributions in the development and deployment of robust assisted activities of daily living system in healthcare sector. Details regarding various sensors, deployment, configuration and performance evaluation have been discussed, alongside outstanding challenges encountered when the technologies are deployed in realistic environments. The researchers should potentially, focus on multidisciplinary work, involving members from the public as subjects and combine expertise from different research fields which might lay the foundation for a more realistic system rather than just relying on a groundtruth device use for validation of different application RF sensing technologies in healthcare domain.

\section{REFERENCES}

[1] J. Lloret, A. Canovas, S. Sendra, and L. Parra, "A smart communication architecture for ambient assisted living," IEEE Commun. Mag., vol. 53, no. 1, pp. 26-33, 2015.

[2] J. Wan, C. A. Byrne, M. J. O'Grady, and G. M. P. O'Hare, "Managing Wandering Risk in People With Dementia," IEEE Trans. HumanMachine Syst., vol. 45, no. 6, pp. 819-823, 2015.

[3] X. Yang et al., "Freezing of Gait Detection Considering Leaky Wave Cable," IEEE Trans. Antennas Propag., vol. 67, no. 1, pp. 554-561, 2019.

[4] S. V. and M. R.B., "Development of Bayesian belief network model for electrical load demand," Int. J. Syst. Assur. Eng. Manag., vol. 1, no. 2, pp. 170-177, 2010.

[5] S. Shukri, L. Munirah Kamarudin, and M. Hafiz Fazalul Rahiman, "Device-Free Localization for Human Activity Monitoring," Intell. Video Surveill. [Working Title], 2019.

[6] W. Li, B. Tan, and R. Piechocki, "Passive Radar for Opportunistic Monitoring in E-Health Applications," IEEE J. Transl. Eng. Heal. Med., vol. 6, pp. 1-10, 2018.

[7] M. G. Amin, Y. D. Zhang, F. Ahmad, and K. C. D. Ho, "Radar Signal Processing for Elderly Fall Detection: The future for in-home monitoring," IEEE Signal Process. Mag., vol. 33, no. 2, pp. 71-80, 2016.

[8] Z. Yang, Z. Zhou, and H. Kong, "25 From RSSI to CSI: Indoor Localization via Channel Response," vol. 46, no. 2, 2013.

[9] A. A. Mehdi, A. Kumar, and A. K. Jaiswal, "A review of RFID-WSN healthcare applications and issues," Ijarece.Org, vol. 6, no. 6, pp. 526-533, 2017.

[10] B. Dong et al., "Monitoring of atopic dermatitis using leaky coaxial cable," Healthc. Technol. Lett., vol. 4, no. 6, pp. 244-248, 2017.

[11] S. Shah et al., "Internet of Things for Sensing: A Case Study in the Healthcare System," Appl. Sci., vol. 8, no. 4, p. 508, 2018.

[12] J. B. Coleman Murray G-Crosby Raymond A Heising T A Hunter H J Reich F E Terman et al., "PROCEEDINGS OF THE I.R.E. (Including WAVES AND ELECTRONS Section) Communication by Means of Reflected Power," Proc. I.R.E. Oct., 1948.

[13] C. G. Caro and J. A. Bloice, "Contactless Apnœa Detector Based on Radar," Lancet, vol. 298, no. 7731, pp. 959-961, 1971.

[14] S. Pisa, E. Pittella, and E. Piuzzi, "A survey of radar systems for medical applications,” IEEE Aerosp. Electron. Syst. Mag., vol. 31, no. 11, pp. 64-81, 2016.

[15] E. Cippitelli, F. Fioranelli, E. Gambi, and S. Spinsante, "Radar and RGB-Depth Sensors for Fall Detection: A Review," IEEE Sens. J., vol. 17, no. 12, pp. 3585-3604, 2017.

[16] B. Jokanovic, M. Amin, and B. Erol, "Multiple joint-variable domains recognition of human motion," in 2017 IEEE Radar Conference (RadarConf), 2017, pp. 948-952.

[17] B. Jokanović and M. Amin, "Suitability of Data Representation Domains in Expressing Human Motion Radar Signals," IEEE Geosci. Remote Sens. Lett., vol. 14, no. 12, pp. 2370-2374, 2017.

[18] F. Lin et al., "SleepSense: A Noncontact and Cost-Effective Sleep Monitoring System," IEEE Trans. Biomed. Circuits Syst., vol. 11, no.
1, pp. 189-202, 2017

[19] A. Rahman, E. Yavari, V. M. Lubecke, and O. Lubecke, "Noncontact Doppler radar unique identification system using neural network classifier on life signs," in 2016 IEEE Topical Conference on Biomedical Wireless Technologies, Networks, and Sensing Systems (BioWireleSS), 2016, pp. 46-48.

[20] J. F. Dolan, “(Received 19 May, 1972),” vol. 17, pp. 472-481, 1972. [21] H. R. Monitoring, M. Lubecke, B. Park, and Q. Zhou, "Signal Processing Methods for Doppler Radar."

[22] W. Massagram, N. M. Hafner, B. Park, V. M. Lubecke, A. HostMadsen, and O. Boric-Lubecke, "Feasibility of Heart Rate Variability Measurement from Quadrature Doppler Radar Using Arctangent Demodulation with DC Offset Compensation," in 2007 29th Annual International Conference of the IEEE Engineering in Medicine and Biology Society, 2007, pp. 1643-1646.

[23] A. D. Droitcour et al., "Non-contact respiratory rate measurement validation for hospitalized patients," in 2009 Annual International Conference of the IEEE Engineering in Medicine and Biology Society, 2009, pp. 4812-4815.

[24] H. Zhao, H. Hong, L. Sun, Y. Li, C. Li, and X. Zhu, "Noncontact Physiological Dynamics Detection Using Low-power Digital-IF Doppler Radar," IEEE Trans. Instrum. Meas., vol. 66, no. 7, pp. 1780-1788, 2017.

[25] H. Zhao et al., "A Noncontact Breathing Disorder Recognition System Using 2.4-GHz Digital-IF Doppler Radar," IEEE J. Biomed. Heal. Informatics, vol. 23, no. 1, pp. 208-217, 2019.

[26] J. E. Kiriazi, O. Boric-Lubecke, and V. M. Lubecke, "Radar cross section of human cardiopulmonary activity for recumbent subject," in 2009 Annual International Conference of the IEEE Engineering in Medicine and Biology Society, 2009, pp. 4808-4811.

[27] M. Baboli, A. Singh, B. Soll, O. Boric-Lubecke, and V. M. Lubecke, "Good Night: Sleep Monitoring Using a Physiological Radar Monitoring System Integrated with a Polysomnography System," IEEE Microw. Mag., vol. 16, no. 6, pp. 34-41, 2015.

[28] A. Singh et al., "Considerations for integration of a physiological radar monitoring system with gold standard clinical sleep monitoring systems," in 2013 35th Annual International Conference of the IEEE Engineering in Medicine and Biology Society (EMBC), 2013, pp. 2120-2123.

[29] E. Yavari, H. Jou, V. Lubecke, and O. Boric-Lubecke, "Doppler radar sensor for occupancy monitoring," in 2013 IEEE Topical Conference on Power Amplifiers for Wireless and Radio Applications, 2013, pp. 145-147.

[30] M. S. Seyfioğlu, A. Serinöz, A. M. Özbayoğlu, and S. Z. Gürbüz, "Feature diverse hierarchical classification of human gait with $\mathrm{CW}$ radar for assisted living," in International Conference on Radar Systems (Radar 2017), 2017, pp. 1-5.

[31] B. Çağlıyan, C. Karabacak, and S. Z. Gürbüz, "Indoor human activity recognition using BumbleBee radar," in 2014 22nd Signal Processing and Communications Applications Conference (SIU), 2014, pp. $1055-1058$.

[32] A. K. Seifert, L. Schäfer, M. G. Amin, and A. M. Zoubir, "Subspace Classification of Human Gait Using Radar Micro-Doppler Signatures," in 2018 26th European Signal Processing Conference (EUSIPCO), 2018, pp. 311-315.

[33] B. Jokanović and M. Amin, "Fall Detection Using Deep Learning in Range-Doppler Radars," IEEE Trans. Aerosp. Electron. Syst., vol. 54, no. 1, pp. 180-189, 2018.

[34] B. Erol and M. G. Amin, "Radar Data Cube Analysis for Fall Detection," in 2018 IEEE International Conference on Acoustics, Speech and Signal Processing (ICASSP), 2018, pp. 2446-2450.

[35] H. Li, A. Shrestha, H. Heidari, J. L. Kernec, and F. Fioranelli, “A Multisensory Approach for Remote Health Monitoring of Older People," IEEE J. Electromagn. RF Microwaves Med. Biol., vol. 2, no. 2, pp. 102-108, 2018.

[36] L. Yang, G. Li, M. Ritchie, F. Fioranelli, and H. Griffiths, "Gait classification based on micro-Doppler features," 2016 CIE Int. Conf. Radar, RADAR 2016, no. October, pp. 10-13, 2017.

[37] Q. Zhang, D. Haider, W. Wang, S. Shah, X. Yang, and Q. Abbasi, "Chronic Obstructive Pulmonary Disease Warning in the Approximate Ward Environment," Appl. Sci., vol. 8, no. 10, p. 1915, 2018.

[38] S. A. K. Tanoli, M. I. Khan, Q. Fraz, X. Yang, and S. A. Shah, "A Compact Beam-Scanning Leaky-Wave Antenna With Improved Performance," IEEE Antennas Wirel. Propag. Lett., vol. 17, no. 5, pp. 
825-828, 2018.

[39] X. Yang et al., "Wandering Pattern Sensing at S-Band," IEEE J. Biomed. Heal. Informatics, vol. 22, no. 6, pp. 1863-1870, 2018.

[40] L. Liu, S. Shah, G. Zhao, and X. Yang, "Respiration Symptoms Monitoring in Body Area Networks," Appl. Sci., vol. 8, no. 4, p. 568, 2018.

[41] S. A. Shah et al., "Buried Object Sensing Considering Curved Pipeline," IEEE Antennas Wirel. Propag. Lett., vol. 16, pp. 2771$2775,2017$.

[42] J. Dunn, "Mini mobile phones are popular among Irish prisoners who hide them 'internally' from guards | Daily Mail Online," Dly. Mail, pp. $485-486,2016$.

[43] S. A. K. Tanoli et al., "An experimental channel capacity analysis of cooperative networks using Universal Software Radio Peripheral (USRP)," Sustain., vol. 10, no. 6, 2018.

[44] X. Yang et al., "Monitoring of Patients Suffering From REM Sleep Behavior Disorder," IEEE J. Electromagn. RF Microwaves Med. Biol., vol. 2, no. 2, pp. 138-143, 2018.

[45] K. Ali, A. X. Liu, W. Wang, and M. Shahzad, "Keystroke Recognition Using WiFi Signals," Proc. 21 st Annu. Int. Conf. Mob. Comput. Netw. - MobiCom '15, pp. 90-102, 2015.

[46] H. Li, W. Yang, J. Wang, Y. Xu, and L. Huang, "WiFinger," Proc. 2016 ACM Int. Jt. Conf. Pervasive Ubiquitous Comput. - UbiComp '16, pp. 250-261, 2016.

[47] Y. Wang, J. Liu, Y. Chen, M. Gruteser, J. Yang, and H. Liu, "E-eyes: device-free location-oriented activity identification using finegrained WiFi signatures," MobiCom '14 Proc. 20th Annu. Int. Conf. Mob. Comput. Netw., pp. 617-628, 2014.

[48] X. Yang et al., "S-Band Sensing-Based Motion Assessment Framework for Cerebellar Dysfunction Patients," IEEE Sens. J., p. 1, 2019.

[49] X. Yang et al., "Detection of Essential Tremor at the S-Band," IEEE J. Transl. Eng. Heal. Med., vol. 6, 2018.

[50] D. Fan et al., "Breathing Rhythm Analysis in Body Centric Networks," IEEE Access, vol. 6, pp. 32507-32513, 2018.

[51] S. A. Shah, D. Fan, A. Ren, N. Zhao, X. Yang, and S. A. K. Tanoli, "Seizure episodes detection via smart medical sensing system," $J$. Ambient Intell. Humaniz. Comput., vol. 0, no. 0, pp. 1-13, 2018.

[52] D. Haider et al., "An efficient monitoring of eclamptic seizures in wireless sensors networks," Comput. Electr. Eng., vol. 75, pp. 16-30, 2019.

[53] X. Yang et al., "Diagnosis of the Hypopnea syndrome in the early stage," Neural Comput. Appl., vol. 8, no. 1, 2019.

[54] D. Haider et al., "Utilizing a 5G spectrum for health care to detect the tremors and breathing activity for multiple sclerosis," Transactions on Emerging Telecommunications Technologies, vol. 29, no. 10. 2018.

[55] M. B. Khan et al., "Design of Software Defined Radios Based Platform for Activity Recognition," IEEE Access, vol. 7, pp. 3108331088, 2019.

[56] W. Alliance and F. O. R. Patient, "WHO PATIENT SAFETY CURRICULUM GUIDE FOR MEDICAL SCHOOLS WORLD ALLIANCE FOR PATIENT SAFETY Curriculum Guide: a summary."

[57] H. J. Yazici, "An exploratory analysis of hospital perspectives on real time information requirements and perceived benefits of RFID technology for future adoption," Int. J. Inf. Manage., vol. 34, no. 5, pp. 603-621, 2014.

[58] W. Yao, C. H. Chu, and Z. Li, "The adoption and implementation of RFID technologies in healthcare: A literature review," J. Med. Syst., vol. 36, no. 6, pp. 3507-3525, 2012.

[59] S. Ajami and A. Rajabzadeh, "Radio Frequency Identification (RFID) technology and patient safety," J. Res. Med. Sci., vol. 18, no. 9, pp. 809-813, Sep. 2013.

[60] M. Friesen, "a Review of Emerging Smart Rfid in," C. Vancouver, $B C$..., no. October, 2014.

[61] S. F. Tzeng, W. H. Chen, and F. Y. Pai, "Evaluating the business value of RFID: Evidence from five case studies," Int. J. Prod. Econ., vol. 112, no. 2, pp. 601-613, 2008.

[62] Y.-T. Liao, T.-L. Chen, T.-S. Chen, Z.-H. Zhong, and J.-H. Hwang, "The Application of RFID to Healthcare Management of Nursing House," Wirel. Pers. Commun., vol. 91, no. 3, pp. 1237-1257, 2016. E. Al-Masri and M. Hamdi, "RFID-Based Approach for Monitoring Patient's Health Inside Hospitals BT - New Trends in Networking, Computing, E-learning, Systems Sciences, and Engineering,” 2015, pp. 607-613.

R. Hornyak, M. Lewis, and B. Sankaranarayan, "Radio frequency identification-enabled capabilities in a healthcare context: An exploratory study," Health Informatics J., vol. 22, no. 3, pp. 562-578, Mar. 2015.

[65] B. Alsinglawi, T. Liu, Q. V. Nguyen, U. Gunawardana, A. Maeder, and S. Simoff, "Passive RFID localisation framework in smart homes healthcare settings," Stud. Health Technol. Inform., vol. 231, pp. 18, 2016.

[66] A. Aguilar, W. Van Der Putten, and G. Maguire, "Positive Patient Identification using RFID and Wireless Networks," Proc. HISI 11th Annu. Conf. Sci. Symp., 2006.

[67] M. Martínez Pérez et al., "Application of RFID Technology in Patient Tracking and Medication Traceability in Emergency Care," J. Med. Syst., vol. 36, no. 6, pp. 3983-3993, 2012.

[68] S. F. Khan, "Health care monitoring system in Internet of Things (IoT) by using RFID," in 2017 6th International Conference on Industrial Technology and Management (ICITM), 2017, pp. 198204.

[69] L. Catarinucci et al., "An IoT-Aware Architecture for Smart Healthcare Systems," IEEE Internet Things J., vol. 2, no. 6, pp. 515526, 2015.

[70] A. S. Atkins and N. Alharbe, "Sensor Technologies using ZigBee and RFID within the Cloud of Internet of Things in Healthcare Applications," Tech. - Int. J. Comput. Sci. Commun. Technol., vol. 6, no. 2, pp. 923-928, 2014.

[71] S. Amendola, R. Lodato, S. Manzari, C. Occhiuzzi, and G. Marrocco, "RFID Technology for IoT-Based Personal Healthcare in Smart Spaces," IEEE Internet Things J., vol. 1, no. 2, pp. 144-152, 2014.

[72] H. Q. Omar, A. Khoshnaw, and W. Monnet, "Smart patient management, monitoring and tracking system using radio-frequency identification (RFID) technology," in 2016 IEEE EMBS Conference on Biomedical Engineering and Sciences (IECBES), 2016, pp. 4045.

[73] S. A. Vora et al., "On implementing an unconventional infant vital signs monitor with passive RFID tags," in 2017 IEEE International Conference on RFID (RFID), 2017, pp. 47-53.

[74] S.-H. Cheng, "An intelligent infant healthcare system of vital signs integrated by active RFID," in 2013 International Conference on Machine Learning and Cybernetics, 2013, vol. 03, pp. 1157-1160.

[75] L. Zhu, R. Wang, Z. Wang, and H. Yang, "TagCare: Using RFIDs to Monitor the Status of the Elderly Living Alone," IEEE Access, vol. 5, pp. 11364-11373, 2017.

[76] A. Schwarzmeier, R. Weigel, G. Fischer, and D. Kissinger, "A low power fall detection and activity monitoring system for nursing facilities and hospitals," in 2014 IEEE Topical Conference on Biomedical Wireless Technologies, Networks, and Sensing Systems (BioWireleSS), 2014, pp. 28-30.

[77] H. Kamoi, K. Toyoda, and T. Ohtsuki, "Fall Detection Using UHF Passive RFID Based on the Neighborhood Preservation Principle," in 2018 IEEE Global Communications Conference (GLOBECOM), 2018, pp. 1-6.

[78] W. Ruan, L. Yao, Q. Z. Sheng, N. Falkner, and X. Li, "TagTrack: Device-Free Localization and Tracking Using Passive RFID Tags," 2014.

[79] Y. C. Chen and Y. W. Lin, "Indoor RFID gait monitoring system for fall detection," 2010 2nd Int. Symp. Aware Comput. ISAC 2010 Symp. Guid., pp. 207-212, 2010.

[80] B. Kaudki and A. Surve, "IOT Enabled Human Fall Detection Using Accelerometer and RFID Technology," 2018 Second Int. Conf. Intell. Comput. Control Syst., no. Iciccs, pp. 1094-1099, 2019.

[81] S. H. Cheng, "An intelligent infant healthcare system of vital signs integrated by active RFID," Proc. - Int. Conf. Mach. Learn. Cybern., vol. 3, pp. 1157-1160, 2013.

[82] S. Lermthong, P. Suwanna, and S. Airphaiboon, "Bedside patient monitoring by NFC," BMEiCON 2016 - 9th Biomed. Eng. Int. Conf., pp. 1-4, 2017.

[83] F. Fioranelli, M. Ritchie, and H. Griffiths, "Centroid features for classification of armed/unarmed multiple personnel using multistatic human micro-Doppler," IET Radar, Sonar Navig., vol. 10, no. 9, pp. 1702-1710, 2016

[84] F. Fioranelli, M. Ritchie, and H. Griffiths, "Multistatic human microDoppler classification of armed/unarmed personnel," IET Radar, Sonar Navig., vol. 9, no. 7, pp. 857-865, 2015.

[85] F. Fioranelli, M. Ritchie, and H. Griffiths, "Analysis of polarimetric 
multistatic human micro-Doppler classification of armed/unarmed personnel," in 2015 IEEE Radar Conference (RadarCon), 2015, pp. 432-437.

[86] F. Fioranelli, M. Ritchie, and H. Griffiths, "Classification of Unarmed/Armed Personnel Using the NetRAD Multistatic Radar for Micro-Doppler and Singular Value Decomposition Features," IEEE Geosci. Remote Sens. Lett., vol. 12, no. 9, pp. 1933-1937, 2015.

F. Fioranelli, M. Ritchie, and H. Griffiths, "Aspect angle dependence and multistatic data fusion for micro-Doppler classification of armed/unarmed personnel," IET Radar, Sonar Navig., vol. 9, no. 9, pp. 1231-1239, 2015.

[88] S. Tomii and T. Ohtsuki, "Falling detection using multiple doppler sensors," in 2012 IEEE 14th International Conference on e-Health Networking, Applications and Services (Healthcom), 2012, pp. 196201.

[89] E. Kyriacou, S. Christofides, and C. S. Pattichis, "XIV mediterranean conference on medical and biological engineering and computing 2016: MEDICON 2016, March 31st-April 2nd 2016, Paphos, Cyprus,"' IFMBE Proc., vol. 57, pp. 859-864, 2016.
[90] B. Hu, K. Y. See, and W.-Y. C. Richard, "Evaluation of ferrite core EMI suppression under realistic working conditions," in 2008 AsiaPacific Symposium on Electromagnetic Compatibility and 19th International Zurich Symposium on Electromagnetic Compatibility, 2008, pp. 774-777.

[91] A. Coustasse, B. Cunningham, S. Deslich, E. Wilson, and P. Meadows, "Management of RFID Systems in Hospital Transfusion Services," Bus. Heal. Adm. Assoc. Annu. Conf., pp. 1-16, 2015.

[92] A. Fakhr, "Security, privacy and ethical barriers in adoption of RFID in healthcare sector (Iranian Hospitals)," Journal of Engineering and Applied Sciences, vol. 11, no. 8. pp. 1811-1821, 2016. 